\title{
Risk-adjustment models for heart failure patients' 30-day mortality and readmission rates: the incremental value of clinical data abstracted from medical charts beyond hospital discharge record
}

\author{
Jacopo Lenzi ${ }^{1 \dagger}$, Vera Maria Avaldi ${ }^{1 \dagger}$, Tina Hernandez-Boussard ${ }^{2}$, Carlo Descovich ${ }^{3}$, Ilaria Castaldini ${ }^{4}$,
} Stefano Urbinati ${ }^{5}$, Giuseppe Di Pasquale ${ }^{6}$, Paola Rucci ${ }^{1}$ and Maria Pia Fantini ${ }^{*^{*}}$

\begin{abstract}
Background: Hospital discharge records (HDRs) are routinely used to assess outcomes of care and to compare hospital performance for heart failure. The advantages of using clinical data from medical charts to improve risk-adjustment models remain controversial. The aim of the present study was to evaluate the additional contribution of clinical variables to HDR-based 30-day mortality and readmission models in patients with heart failure.
\end{abstract}

Methods: This retrospective observational study included all patients residing in the Local Healthcare Authority of Bologna (about 1 million inhabitants) who were discharged in 2012 from one of three hospitals in the area with a diagnosis of heart failure. For each study outcome, we compared the discrimination of the two risk-adjustment models (i.e., HDR-only model and HDR-clinical model) through the area under the ROC curve (AUC).

Results: A total of 1145 and 1025 patients were included in the mortality and readmission analyses, respectively. Adding clinical data significantly improved the discrimination of the mortality model (AUC $=0.84$ vs. $0.73, p<0.001$ ), but not the discrimination of the readmission model ( $A \cup C=0.65$ vs. $0.63, p=0.08$ ).

Conclusions: We identified clinical variables that significantly improved the discrimination of the HDR-only model for 30-day mortality following heart failure. By contrast, clinical variables made little contribution to the discrimination of the HDR-only model for 30-day readmission.

Keywords: Heart failure, Risk-adjustment, Mortality, Readmissions

Abbreviations: AGENAS, Agenzia Nazionale per i Servizi Sanitari Regionali; AIC, Akaike information criterion; AUC, Area under the ROC curve; HDR, Hospital discharge record; HF, Heart failure; ICD, Implantable cardioverter defibrillator; LHA, Local healthcare authority; LR, Likelihood ratio; OPD, Outpatient pharmaceutical database; PNE, Programma Nazionale Esiti; ROC, Receiver operation characteristic

\footnotetext{
* Correspondence: mariapia.fantini@unibo.it

${ }^{\dagger}$ Equal contributors

'Department of Biomedical and Neuromotor Sciences, Alma Mater

Studiorum - University of Bologna, via San Giacomo 12, 40126 Bologna, Italy

Full list of author information is available at the end of the article
} 


\section{Background}

Heart failure (HF) is a complex syndrome characterized by high mortality and morbidity and is a leading cause of hospitalization [1]. Aging population, decreased HF mortality due to improvement of therapeutic interventions, effective secondary prevention, and hospital and primary care management strategies have led to an increased burden of HF on healthcare systems [1, 2].

Assessing quality of care, especially outcomes of care, and comparing hospital performance have become important issues needed to ensure a healthcare system that is cost-effective for HF [3, 4]. To this end, statistical models to compare hospital performance across important outcomes must adjust for differences in demographic and clinical characteristics, as the case mix of patients may vary among regions and hospitals $[5,6]$.

Hospital discharge records (HDRs) (also known as administrative claims databases) are a main source of data for outcomes studies because data collection is inexpensive and they enable the analysis of large populations and a large number of conditions and pathologies [7]; however, these data lack clinical granularity and do not allow one to determine the severity and history of disease [5-8]. In Italy, the National Outcome Evaluation Program (Programma Nazionale Esiti - PNE), an initiative endorsed by the National Agency for Regional Health Services (Agenzia Nazionale per i Servizi Sanitari Regionali-AGENAS) that monitors healthcare outcomes across hospitals and municipalities, routinely uses HDRs to derive hospital-specific indicators and important patient characteristics used in risk-adjustment of different patient populations [9].

Many authors have underlined the advantages of adding specific clinical data to HDR-based risk-adjustment models [8, 10-13]: the integration of clinical data with HDRs in risk-adjustment models could improve the predictive power and the control of confounding, and identify variables that mainly influence the outcome [13]. Medical charts could indeed offer important information on the patient's clinical conditions unavailable in HDRs that allow one to differentiate between comorbidities and complications [8].

With regard to HF, a recent study [14] suggested that the addition of clinical data to HDR-based models improved the discrimination of mortality risk-adjustment models and shifted mortality performance rankings in inter-hospital comparison. However, clinical data did not substantially improve the discrimination of the readmission risk model nor the hospital ranking. Another study [5] found no difference between HDR- and clinical-based predictive models for 30-day mortality. Overall, a recent systematic review of the literature revealed that the discriminatory ability of the models was generally higher for the prediction of death than for the prediction of hospital readmission [15].
The impact of clinical data in risk-adjustment models is therefore controversial because, even though clinical data add information, they do not always improve the discrimination of the models. Moreover, the availability and reliability of clinical data may vary greatly among hospitals, and their collection entails more effort and costs than HDRs data. For this reason, it is useful to identify a limited number of clinical variables that are significantly correlated with HF outcomes and are affordable and easy to collect [7]. The aim of this study was to evaluate the usefulness of clinical variables and drug prescriptions in predicting 30-day mortality and 30-day readmissions in patients with HF.

\section{Methods}

\section{Setting and study population}

This retrospective observational study included all patients residing in the Local Healthcare Authority (LHA) of Bologna who were discharged from one of three public hospitals in the area (hereinafter called " $A$ ", "B" and "C") between December 2, 2011 and December 1, 2012 with a primary diagnosis of HF (ICD-9-CM diagnosis codes: 398.91, 402.x1, 404.x1, 404.x3, 428.xx). Data were retrieved from the HDRs Database (see Additional file 1 for a description of the data source).

Hospital A is the second largest hospital of Bologna, with more than 900 beds and about 40 wards; hospital $\mathrm{B}$, also located in Bologna, has about 370 bed and 20 wards, and is a center of excellence in the field of neuroscience; hospital $\mathrm{C}$ is located in a municipality not far from Bologna, with about 200 beds and 15 wards, and is the referral facility in the northern LHA area.

Patients were excluded if any of the following criteria were met:

1. A secondary diagnosis of non-cardiogenic acute pulmonary edema or acute kidney failure (ICD-9-CM diagnosis codes: $518.4,584 . x)$, i.e., patients with symptoms probably related to causes other than HF, in keeping with the PNE definition to allow comparison of results;

2. Age $<18$ or $>100$ years, because very young and very old patients may have distinctive clinical features at diagnosis and survival;

3. Transfer from another facility, to ascribe the study outcomes to the hospital where the patient was initially admitted;

4. Incomplete clinical data (i.e., missing laboratory data, electrocardiography, etc.), to ensure comparability of risk-adjustment models. We decided to exclude these patients because the pattern of missing clinical data appeared to be independent of patient's age, gender and length of stay (data not shown). 
For patients with multiple eligible hospital admissions over the 1-year study period, we considered only the first one as the index admission because hospitalizations of the same patient are correlated, thus violating the assumption of independence required by regression models. Still, we are aware that excluding multiple readmissions, i.e., hospital admissions of patients with presumably chronic $\mathrm{HF}$, may limit the generalizability of the results.

\section{Data}

Variables considered for inclusion in risk-adjustment models were retrieved from three data sources: (1) HDRs, (2) Outpatient Pharmaceutical Database (OPD), and (3) medical charts (see Additional file 1 for a description of the data sources).

Variables retrieved from the HDRs Database were: age, gender, length of stay, and 23 comorbidities chosen a priori and identified in the index hospitalization and in all hospital admissions occurring two-years prior to the index hospitalization (see Additional file 2, which includes the detailed list of comorbidities). Moreover, we collected from the OPD information on filled prescriptions of: antidiabetic drugs (ATC code A10), drugs for cardiac therapy (C01), drugs for obstructive airway diseases (R03), diuretics (C03), $\beta$-blockers (C07), angiotensin-converting enzyme inhibitors/angiotensin receptor blockers (C09), calcium channel blockers and/or other antihypertensive drugs (C08, C02), statins (C10AA), and antiplatelet drugs (B01AC). Treatment for each drug was defined as at least one filled prescription in the three months preceding the HF hospital admission.

Clinical data abstracted from medical chart review were:

1. Emergency department utilization (yes/no);

2. Heart rate (bpm) and systolic blood pressure (mmHg) at hospital admission;

3. Pulmonary congestion (yes/no), determined with radiography at hospital admission;

4. Heart rhythm (sinus rhythm/atrial fibrillation/ pacemaker rhythm), bundle branch block (no/right/ left), and QRS complex (only for patients with left bundle branch block) retrieved from electrocardiography at hospital admission;

5. Serum creatinine $(\mathrm{mg} / \mathrm{dL})$, sodium $(\mathrm{mmol} / \mathrm{L})$, and hemoglobin $(\mathrm{g} / \mathrm{dL})$ at hospital admission;

6. Previously implanted cardiac devices, including cardiac resynchronization therapies (yes/no): pacemaker, implantable cardioverter defibrillator (ICD).

We selected these clinical data for three main reasons. First, other studies have shown that these data are predictive of mortality and/or readmission among patients with HF [15-21]. Second, most of these measures are quantitative measures that are not captured in HDRs. Lastly these data reflect multiple clinical domains, including laboratory test results, diagnostic test results and vital signs.

The data collection for the present study consisted of a thorough review of more than 1000 medical charts which, in Emilia-Romagna, are still paper-based. This review process was carried out by four medical residents in Public Health who had been previously trained by a team of cardiologists. The objective of this training was to test and improve the review of medical charts, and to ensure that medical residents would collect only clinical data determined at hospital admission, i.e., prior to any medical intervention. Medical residents worked always in pairs and imputed data abstracted from medical charts in a spreadsheet which was later linked with HDRs and OPD using the patient's identification code.

\section{Study outcomes}

The study had two main outcomes of interest. The first outcome was all-cause death within thirty days of index admission, identified through the Regional Mortality Register Database (see Additional file 1 for a description of the data source). The second outcome of interest was all-cause unplanned readmission to any Italian hospital between two to thirty days after the index discharge and lasting more than one day. For the readmission analysis we excluded patients deceased during index hospitalization, as they cannot experience rehospitalizations.

\section{Statistical analysis}

Analysis of variance, Kruskal-Wallis test, $\chi^{2}$ test and Fisher's exact test were used, where appropriate, to compare the distribution of patients' demographic and clinical characteristics across hospitals.

\section{Risk-adjustment models based on HDR variables}

The crude association between each potential predictor and the study outcomes (30-day mortality and unplanned readmissions) was first examined in univariable logistic regression models. In these models, age and length of stay were transformed into normally distributed variables (cubed and log-transformed, respectively) in order to linearize their relationship with the logistic link function [22]. Predictors significantly associated with the outcome at $p<0.25$ in univariable analyses were selected for inclusion in multivariable logistic regression models. A bootstrap procedure was used to determine which of these factors were significantly associated with the outcome in multivariable models. Using this approach, 1000 replicated bootstrap samples were selected from the original cohort. In each replicated sample, age and gender were forced into the model, and a backward 
elimination of potential confounders was applied with a significance level of removal equal to 0.05 . Only risk factors selected in at least $50 \%$ of the replicates were included as covariates in the final multivariable logistic regression models.

\section{Risk-adjustment models based on HDR plus OPD plus medical charts variables}

The steps described above to build the HDR-only model were replicated by adding the variables retrieved from OPD and medical charts to HRD variables. Systolic blood pressure was log-transformed and serum creatinine was transformed using the reciprocal of square root to normalize their distribution.

For each study outcome, we compared the discrimination of the two risk-adjustment models through the area under the ROC curve (AUC). Discrimination is the ability of the model to distinguish between high-risk and low-risk patients. The incremental contribution of the variables retrieved from OPD plus medical charts was assessed also using the likelihood ratio (LR) test, because it has been demonstrated that the AUC test produces conservative test size and low power [23]. Lastly, the goodness of fit of each model was estimated using the coefficient of determination (McFadden's adjusted pseudo $R^{2}$ ) and the Akaike Information Criterion (AIC).

Statistical analyses were carried out using the Stata software package, version 13 (StataCorp. 2013. Stata Statistical Software: Release 13. College Station, TX: StataCorp LP).

\section{Results}

Of the 1334 patients discharged after HF, 1145 (85.8 \%) met inclusion criteria for the mortality analyses, while $1025(76.8 \%)$ met inclusion criteria for the readmission analyses (Fig. 1). The overall 30-day mortality and 30-day readmission rates were $13.2 \%$ (151 patients out of 1145) and $15.6 \%$ (160 patients out of 1025), respectively. Of the 151 deaths within 30 days of hospital admission, 15 (9.9\%) occurred on the day of admission, and 37 (24.5\%) occurred after hospital discharge. Of the 160 patients rehospitalized within 30 days of discharge, 55 (34.4\%) were readmitted to the hospital for another episode of HF, and 21 (13.1\%) for cardiovascular causes other than HF.

The crude 30-day mortality rates in hospitals A, B and C were $16.0,2.1$ and $8.7 \%$, while the crude 30 -day readmission rates were 15.2, 24.2 and $13.5 \%$, respectively.

The distribution of patient characteristics, overall and by hospital, is reported in Table 1 . Mean age was 81 years, $54.3 \%$ were female, and median length of stay was 7 days. The most frequent comorbid conditions were hypertension (32.7\%), other forms of ischemic heart disease (32.4\%), previous HF diagnosis (28.9\%), conduction disorders and cardiac dysrhythmias (27.7\%), and chronic nephropathies (26.6\%). Mean heart rate and systolic blood pressure were $87 \pm 20.2 \mathrm{bpm}$ and $140 \pm$ $32.6 \mathrm{mmHg}$, respectively. Almost two thirds of patients (60.7\%) had used calcium channel blockers and/or other antihypertensive drugs before admission. Some differences in the case mix of patients discharged from the three hospitals were found; specifically, patients from hospital A were older and more frequently female, and had more

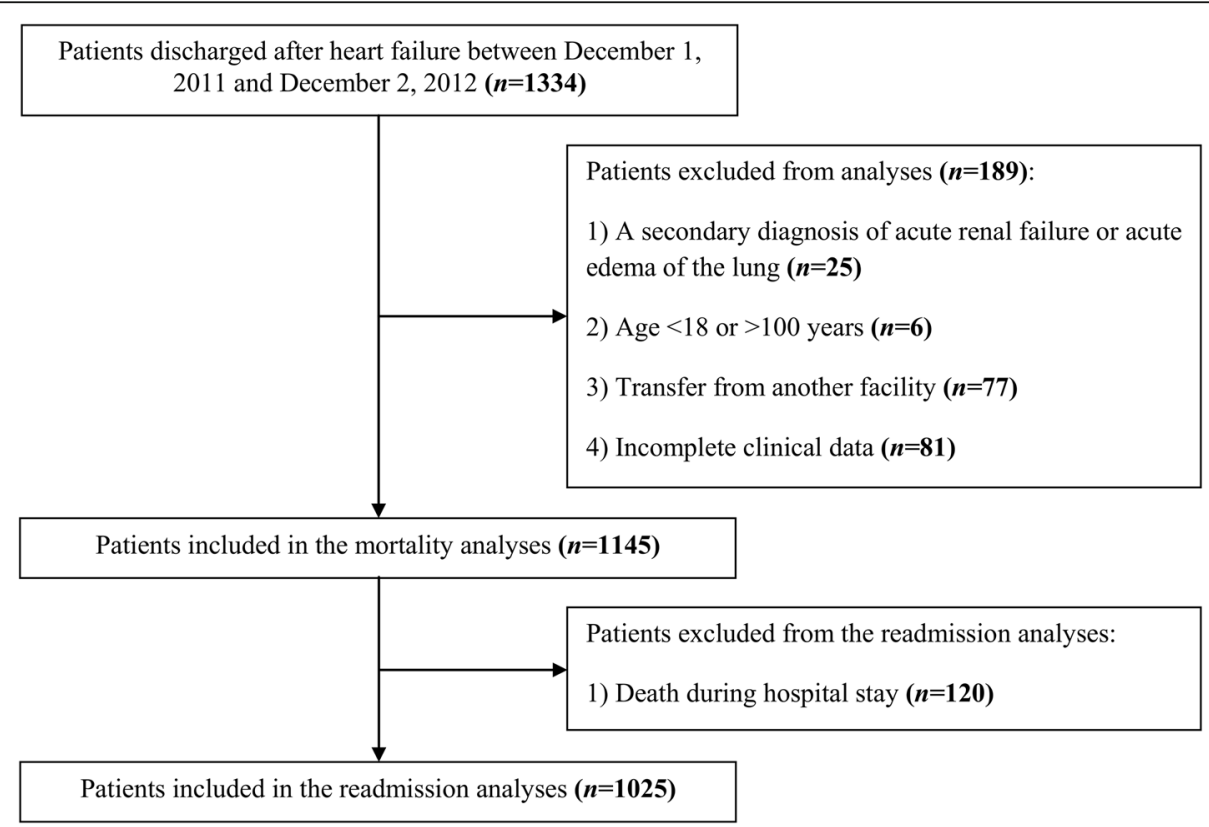

Fig. 1 Patients' flow diagram 
Table 1 Distribution of patient characteristics collected from HDRs, OPD and medical charts, overall and by hospital

\begin{tabular}{|c|c|c|c|c|c|c|c|c|c|}
\hline \multirow{3}{*}{$\begin{array}{l}\text { Patient characteristics } \\
\text { From HDRs }\end{array}$} & \multirow{2}{*}{\multicolumn{2}{|c|}{$\frac{\text { Hospital A }}{(n=786)}$}} & \multirow{2}{*}{\multicolumn{2}{|c|}{$\frac{\text { Hospital B }}{(n=96)}$}} & \multirow{2}{*}{\multicolumn{2}{|c|}{$\frac{\text { Hospital C }}{(n=263)}$}} & \multirow{2}{*}{\multicolumn{2}{|c|}{$\frac{\text { All }}{(n=1145)}$}} & \multirow[t]{3}{*}{$p$} \\
\hline & & & & & & & & & \\
\hline & \multirow{3}{*}{82.3} & \multirow{3}{*}[9.2]{} & \multirow{3}{*}{77.1} & \multirow{3}{*}{ [11.0] } & \multirow{3}{*}{79.7} & \multirow{3}{*}{ [10.2] } & \multirow{3}{*}{81.3} & \multirow{3}{*}[9.8]{} & \\
\hline Age in years, mean [SD] & & & & & & & & & $<0.001$ \\
\hline Gender, n (\%) & & & & & & & & & 0.01 \\
\hline Male & 339 & $(43.1)$ & 55 & $(57.3)$ & 129 & $(49.0)$ & 523 & $(45.7)$ & \\
\hline Female & 447 & $(56.9)$ & 41 & $(42.7)$ & 134 & $(51.0)$ & 622 & $(54.3)$ & \\
\hline Length of stay in days, median [IQR] & 8 & {$[7]$} & 9.5 & {$[9.5]$} & 8 & {$[6]$} & 8 & [7] & $<0.001$ \\
\hline \multicolumn{10}{|l|}{ Comorbidity, n (\%) } \\
\hline Malignant tumors & 75 & $(9.5)$ & 11 & $(11.5)$ & 31 & $(11.8)$ & 117 & $(10.2)$ & 0.53 \\
\hline Diabetes & 129 & $(16.4)$ & 19 & $(19.8)$ & 49 & $(18.6)$ & 197 & $(17.2)$ & 0.56 \\
\hline Disorders of lipoid metabolism & 44 & $(5.6)$ & 6 & $(6.3)$ & 27 & $(10.3)$ & 77 & $(6.7)$ & 0.03 \\
\hline Obesity & 23 & $(2.9)$ & 2 & $(2.1)$ & 13 & $(4.9)$ & 38 & (3.3) & 0.26 \\
\hline Hematologic diseases & 127 & $(16.2)$ & 10 & $(10.4)$ & 50 & $(19.0)$ & 187 & $(16.3)$ & 0.14 \\
\hline Hypertensive diseases & 247 & $(31.4)$ & 31 & $(32.3)$ & 96 & $(36.5)$ & 374 & $(32.7)$ & 0.31 \\
\hline Previous AMI & 122 & $(15.5)$ & 25 & $(26.0)$ & 48 & $(18.3)$ & 195 & $(17.0)$ & 0.03 \\
\hline Other forms of ischemic heart disease & 257 & $(32.7)$ & 42 & $(43.8)$ & 72 & $(27.4)$ & 371 & $(32.4)$ & 0.01 \\
\hline III-defined descriptions and complications of heart disease & 12 & $(1.5)$ & 4 & $(4.2)$ & 6 & $(2.3)$ & 22 & $(1.9)$ & 0.14 \\
\hline Rheumatic heart disease & 50 & $(6.4)$ & 9 & $(9.4)$ & 15 & $(5.7)$ & 74 & $(6.5)$ & 0.45 \\
\hline Cardiomyopathies & 56 & $(7.1)$ & 21 & $(21.9)$ & 19 & $(7.2)$ & 96 & $(8.4)$ & $<0.001$ \\
\hline Other cardiac diseases & 60 & $(7.6)$ & 21 & $(21.9)$ & 34 & $(12.9)$ & 115 & $(10.0)$ & $<0.001$ \\
\hline Conduction disorders and cardiac dysrhythmias & 207 & $(26.3)$ & 30 & $(31.3)$ & 80 & $(30.4)$ & 317 & $(27.7)$ & 0.32 \\
\hline Cerebrovascular diseases & 121 & $(15.4)$ & 21 & $(21.9)$ & 50 & $(19.0)$ & 192 & $(16.8)$ & 0.15 \\
\hline Vascular diseases & 66 & $(8.4)$ & 11 & $(11.5)$ & 26 & $(9.9)$ & 103 & $(9.0)$ & 0.52 \\
\hline COPD & 122 & $(15.5)$ & 19 & $(19.8)$ & 42 & $(16.0)$ & 183 & $(16.0)$ & 0.56 \\
\hline Chronic nephropathies & 173 & $(22.0)$ & 34 & $(35.4)$ & 97 & $(36.9)$ & 304 & (26.6) & $<0.001$ \\
\hline Chronic diseases of liver, pancreas and intestine & 16 & $(2.0)$ & 1 & $(1.0)$ & 5 & $(1.9)$ & 22 & $(1.9)$ & $>0.99$ \\
\hline Previous bypass & 14 & $(1.8)$ & 6 & $(6.3)$ & 18 & $(6.8)$ & 38 & (3.3) & $<0.001$ \\
\hline Previous $\mathrm{PCl}$ & 40 & $(5.1)$ & 9 & $(9.4)$ & 16 & $(6.1)$ & 65 & $(5.7)$ & 0.20 \\
\hline Other surgery of the heart & 18 & $(2.3)$ & 9 & $(9.4)$ & 7 & $(2.7)$ & 34 & $(3.0)$ & $<0.01$ \\
\hline Other surgery of great vessels & 18 & $(2.3)$ & 2 & $(2.1)$ & 8 & $(3.0)$ & 28 & $(2.4)$ & 0.74 \\
\hline Previous diagnosis of heart failure & 223 & $(28.4)$ & 34 & $(35.4)$ & 74 & $(28.1)$ & 331 & $(28.9)$ & 0.34 \\
\hline \multicolumn{10}{|l|}{ From OPD } \\
\hline \multicolumn{10}{|l|}{ Previous medication use, $\mathrm{n}(\%)$} \\
\hline Antidiabetic drugs & 174 & $(22.1)$ & 24 & $(25.0)$ & 59 & $(22.4)$ & 257 & $(22.4)$ & 0.79 \\
\hline Drugs for cardiac therapy & 262 & $(33.3)$ & 25 & $(26.0)$ & 70 & $(26.6)$ & 357 & $(31.2)$ & 0.07 \\
\hline Drugs for obstructive airway diseases & 163 & $(20.7)$ & 30 & $(31.3)$ & 70 & $(26.6)$ & 263 & $(23.0)$ & 0.02 \\
\hline Diuretics & 457 & $(58.1)$ & 59 & $(61.5)$ & 159 & $(60.5)$ & 675 & $(59.0)$ & 0.70 \\
\hline$\beta$-blockers & 200 & $(25.4)$ & 32 & $(33.3)$ & 75 & $(28.5)$ & 307 & $(26.8)$ & 0.20 \\
\hline ACEIs/ARBs & 366 & $(46.6)$ & 38 & $(39.6)$ & 115 & $(43.7)$ & 519 & $(45.3)$ & 0.36 \\
\hline Calcium channel blockers and/or other antihypertensive drugs & 472 & $(60.1)$ & 68 & $(70.8)$ & 155 & $(58.9)$ & 695 & $(60.7)$ & 0.10 \\
\hline Statins & 383 & $(48.7)$ & 53 & $(55.2)$ & 131 & $(49.8)$ & 567 & $(49.5)$ & 0.49 \\
\hline Antiplatelet drugs & 181 & $(23.0)$ & 25 & $(26.0)$ & 56 & $(21.3)$ & 262 & $(22.9)$ & 0.63 \\
\hline \multicolumn{10}{|l|}{ From medical charts } \\
\hline Emergency department utilization, n (\%) & 767 & $(97.6)$ & 78 & $(81.3)$ & 256 & $(97.3)$ & 1101 & $(96.2)$ & $<0.001$ \\
\hline
\end{tabular}


Table 1 Distribution of patient characteristics collected from HDRs, OPD and medical charts, overall and by hospital (Continued)

\begin{tabular}{|c|c|c|c|c|c|c|c|c|c|}
\hline Heart rate in bpm, mean $[S D]^{a}$ & 87 & {$[20.7]$} & 86 & [19.4] & 85 & {$[18.7]$} & 87 & {$[20.2]$} & 0.52 \\
\hline Systolic blood pressure in mmHg, mean [SD] & 140 & {$[32.8]$} & 139 & {$[32.5]$} & 142 & {$[32.3]$} & 140 & {$[32.6]$} & 0.67 \\
\hline Pulmonary congestion, $\mathrm{n}(\%)$ & 666 & $(84.7)$ & 87 & (90.6) & 241 & $(91.6)$ & 994 & $(86.8)$ & $<0.001$ \\
\hline Heart rhythm, n (\%) & & & & & & & & & 0.14 \\
\hline Sinus rhythm & 405 & $(51.5)$ & 42 & (43.8) & 127 & $(48.3)$ & 574 & $(50.1)$ & \\
\hline Atrial fibrillation & 323 & $(41.1)$ & 41 & $(42.7)$ & 118 & $(44.9)$ & 482 & $(42.1)$ & \\
\hline Pacemaker rhythm & 55 & (7.0) & 13 & $(13.5)$ & 18 & $(6.8)$ & 86 & $(7.5)$ & \\
\hline Bundle branch block, n (\%) & & & & & & & & & 0.55 \\
\hline No & 625 & (79.5) & 78 & $(81.3)$ & 208 & $(79.1)$ & 911 & $(79.6)$ & \\
\hline Right & 72 & (9.2) & 6 & (6.3) & 30 & $(11.4)$ & 108 & $(9.4)$ & \\
\hline Left & 89 & $(11.3)$ & 12 & (12.5) & 25 & $(9.5)$ & 126 & $(11.0)$ & \\
\hline QRS complex, mean $[S D]^{b}$ & 151 & [18.1] & 159 & [24.0] & 152 & [19.9] & 152 & {$[19.0]$} & 0.25 \\
\hline Serum creatinine in $\mathrm{mg} / \mathrm{dL}$, mean $[\mathrm{SD}]$ & 1.42 & {$[0.9]$} & 1.32 & {$[0.6]$} & 1.4 & {$[0.9]$} & 1.41 & {$[0.9]$} & 0.59 \\
\hline Chronic kidney disease stage (using MDRD formula), n (\%) & & & & & & & & & 0.38 \\
\hline 1 & 60 & (7.6) & 9 & (9.4) & 32 & $(12.2)$ & 101 & $(8.8)$ & \\
\hline 2 & 210 & $(26.7)$ & 25 & (26.0) & 74 & $(28.1)$ & 309 & $(27.0)$ & \\
\hline $3 a$ & 191 & $(24.3)$ & 24 & $(25.0)$ & 53 & $(20.2)$ & 268 & $(23.4)$ & \\
\hline $3 b$ & 179 & (22.8) & 27 & $(28.1)$ & 60 & $(22.8)$ & 266 & $(23.2)$ & \\
\hline 4 & 122 & $(15.5)$ & 10 & $(10.4)$ & 35 & $(13.3)$ & 167 & $(14.6)$ & \\
\hline 5 & 24 & (3.1) & 1 & $(1.0)$ & 9 & (3.4) & 34 & $(3.0)$ & \\
\hline Sodium in $\mathrm{mmol} / \mathrm{l}$, mean $[\mathrm{SD}]$ & 139 & {$[5.8]$} & 139 & [3.7] & 138 & {$[4.8]$} & 139 & {$[5.4]$} & $<0.001$ \\
\hline Hemoglobin in $\mathrm{g} / \mathrm{dL}$, mean $[\mathrm{SD}]$ & 12.1 & [2.2] & 12.1 & [1.9] & 12.1 & {$[2.0]$} & 12.1 & {$[2.1]$} & $>0.90$ \\
\hline Previously implanted pacemaker, n (\%) & 80 & $(10.2)$ & 21 & (21.9) & 29 & $(11.0)$ & 130 & $(11.4)$ & $<0.01$ \\
\hline Previously implanted cardioverter defibrillator, n (\%) & 13 & (1.7) & 10 & $(10.4)$ & 9 & $(3.4)$ & 32 & $(2.8)$ & $<0.001$ \\
\hline
\end{tabular}

often chronic nephropathies and less often pulmonary congestion; on the other hand, patients discharged from hospital B had more cardiovascular comorbidities and previous device implantation, and used less frequently emergency department.

\section{Comparison of risk-adjustment models}

Table 2 reports the variables retained in the riskadjustment models based on HDRs only (models \#1) and HDR plus clinical data (models \#2). Figure 2 illustrates, for each of the continuous variables retained in models \#2, the predicted probabilities of death and readmission.

\section{Thirty-day mortality}

The probability of 30-day mortality started increasing after the age of 70-80, with no gender differences. Of the HDR variables, only cerebrovascular disease was included in the final model (odds ratio $=1.96, p<0.001$ ). Of the variables retrieved from medical charts, atrial fibrillation and sinus rhythm with a heart rate $\geq 90 \mathrm{bpm}$ were associated with a higher risk of mortality, as well as low systolic blood pressure at hospital admission. Serum creatinine levels between 0.8 and $1.1 \mathrm{mg} / \mathrm{dL}$ were associated with a lower risk of mortality. The inclusion of information from medical charts significantly improved the discrimination of the model (AUC $=0.84$ vs. $0.73, p<0.001$; LR $\left.\chi^{2}=122.41, p<0.001\right)$ and its goodness of fit (pseudo $R^{2}=0.22$ vs. $0.08, \mathrm{AIC}=691.37$ vs. 793.56). Figure 3 illustrates ROC curves for models \#1 and \#2.

\section{Thirty-day readmission}

Older age, longer hospital stay, a history of acute myocardial infarction and low systolic blood pressure levels at hospital admission were associated with a higher risk of 30-day readmission, while gender was unrelated to the outcome. The inclusion of systolic blood pressure did not improve the discrimination $(\mathrm{AUC}=0.65$ vs. 0.63 , $\left.p=0.08 ; \operatorname{LR} \chi^{2}=7.91, p=0.02\right)$ and the goodness of fit of 
Table 2 Odds ratios (ORs) of 30-day mortality and readmission for each of the variables retained in multivariable risk-adjustment models based on HDRs only (\#1) and HDR plus clinical data (\#2)

\begin{tabular}{|c|c|c|c|c|c|c|}
\hline \multirow[t]{2}{*}{ Characteristics } & \multicolumn{3}{|c|}{ Model \#1 } & \multicolumn{3}{|c|}{ Model \#2 } \\
\hline & $\overline{O R}$ & $95 \% \mathrm{Cl}$ & $P$ & $\overline{\mathrm{OR}}$ & $95 \% \mathrm{Cl}$ & $p$ \\
\hline \multicolumn{7}{|l|}{ 30-day mortality } \\
\hline \multicolumn{7}{|l|}{ From HDRs } \\
\hline \multicolumn{7}{|l|}{ Gender } \\
\hline Male & 1.00 & & & 1.00 & & \\
\hline Female & 0.58 & $0.41-0.83$ & $<0.01$ & 0.63 & $0.37-1.06$ & 0.08 \\
\hline $\mathrm{Age}^{\mathrm{a}}$ & 1.00 & $1.00-1.00$ & $<0.001$ & 1.00 & $1.00-1.00$ & $<0.001$ \\
\hline \multicolumn{7}{|l|}{ Cerebrovascular disease } \\
\hline No & 1.00 & & & 1.00 & & \\
\hline Yes & 1.89 & $1.53-2.35$ & $<0.001$ & 1.96 & $1.33-2.87$ & $<0.01$ \\
\hline \multicolumn{7}{|l|}{ From medical charts } \\
\hline Systolic blood pressure ${ }^{\mathrm{b}}$ & - & & & 0.05 & $0.02-0.13$ & $<0.001$ \\
\hline \multicolumn{7}{|l|}{ Heart rhythm } \\
\hline Sinus rhythm with heart rate $<90$ bpm $^{c}$ & - & & & 1.00 & & \\
\hline Sinus rhythm with heart rate $\geq 90$ bpm $^{c}$ & - & & & 2.94 & $1.87-4.61$ & $<0.001$ \\
\hline Atrial fibrillation & - & & & 1.78 & $1.31-2.41$ & $<0.001$ \\
\hline Pacemaker rhythm & - & & & 1.21 & $0.29-5.11$ & 0.79 \\
\hline Serum creatinine ${ }^{d}$ & - & & & 0.26 & $0.12-0.54$ & $<0.001$ \\
\hline \multicolumn{7}{|l|}{ 30-day readmission } \\
\hline \multicolumn{7}{|l|}{ From HDRs } \\
\hline \multicolumn{7}{|l|}{ Gender } \\
\hline Male & 1.00 & & & 1.00 & & \\
\hline Female & 0.95 & $0.66-1.36$ & 0.78 & 0.96 & $0.67-1.38$ & 0.82 \\
\hline $\mathrm{Age}^{\mathrm{a}}$ & 1.00 & $1.00-1.00$ & $<0.01$ & 1.00 & $1.00-1.00$ & $<0.01$ \\
\hline Length of stay ${ }^{\mathrm{b}}$ & 1.41 & $1.14-1.74$ & $<0.01$ & 1.38 & $1.12-1.71$ & $<0.01$ \\
\hline \multicolumn{7}{|l|}{ Previous acute myocardial infarction } \\
\hline No & 1.00 & & & 1.00 & & \\
\hline Yes & 2.05 & $1.11-3.78$ & 0.02 & 1.90 & $1.05-3.44$ & 0.03 \\
\hline \multicolumn{7}{|l|}{ From medical charts } \\
\hline Systolic blood pressure ${ }^{\mathrm{b}}$ & - & & & 0.33 & $0.19-0.59$ & $<0.001$ \\
\hline
\end{tabular}

Abbreviations: $\mathrm{Cl}$ confidence interval

${ }^{\text {a }}$ This variable was cubed to achieve normality

${ }^{\mathrm{b}}$ This variable was log-transformed to achieve normality

'This cutoff was chosen using ROC analysis to minimize the number of false positive and false negatives

${ }^{\mathrm{d}}$ This variable was transformed using the reciprocal of square root to achieve normality. We also included a squared term in the regression to achieve a good

model specification $(\mathrm{OR}=357.41,95 \% \mathrm{Cl}=36.36-3513.66, p<0.001)$

the model (pseudo $R^{2}=0.04$ vs. 0.03 , AIC $=854.95$ vs. 862.86). Figure 4 illustrates ROC curves for models \#1 and \#2.

None of the data on filled prescriptions were retained in the final risk-adjustment models of 30-day mortality and readmission.

\section{Discussion}

In this large, comprehensive, regional study of patients with HF across three hospitals, our results indicate that adding specific clinical variables retrieved from medical charts significantly contributed to the discrimination of HDR-only models for 30-day mortality, but not for 30-day readmissions. These results confirm the findings of other studies that highlighted the benefits of extracting detailed information from medical records to enhance the discrimination of models for 30-day mortality $[8,10,12,14,15,24]$.

Consistent with previous studies [20, 25-34], we found important clinical variables that were significantly associated 
30-day mortality
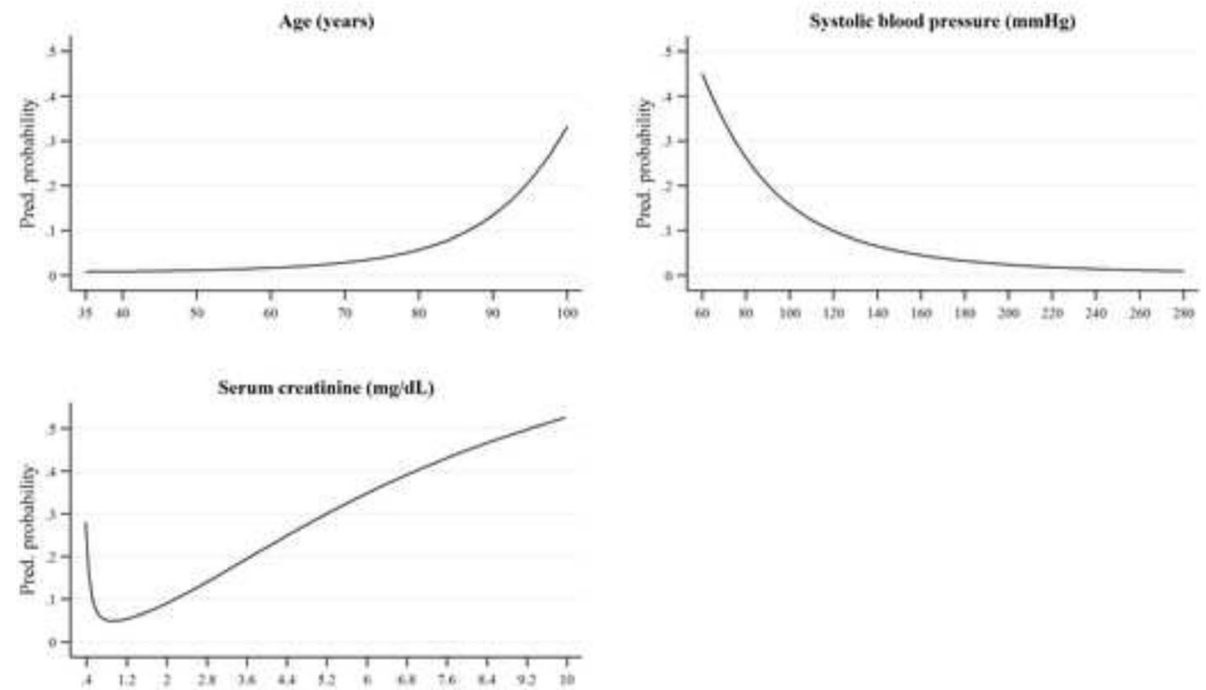

30-day readmission
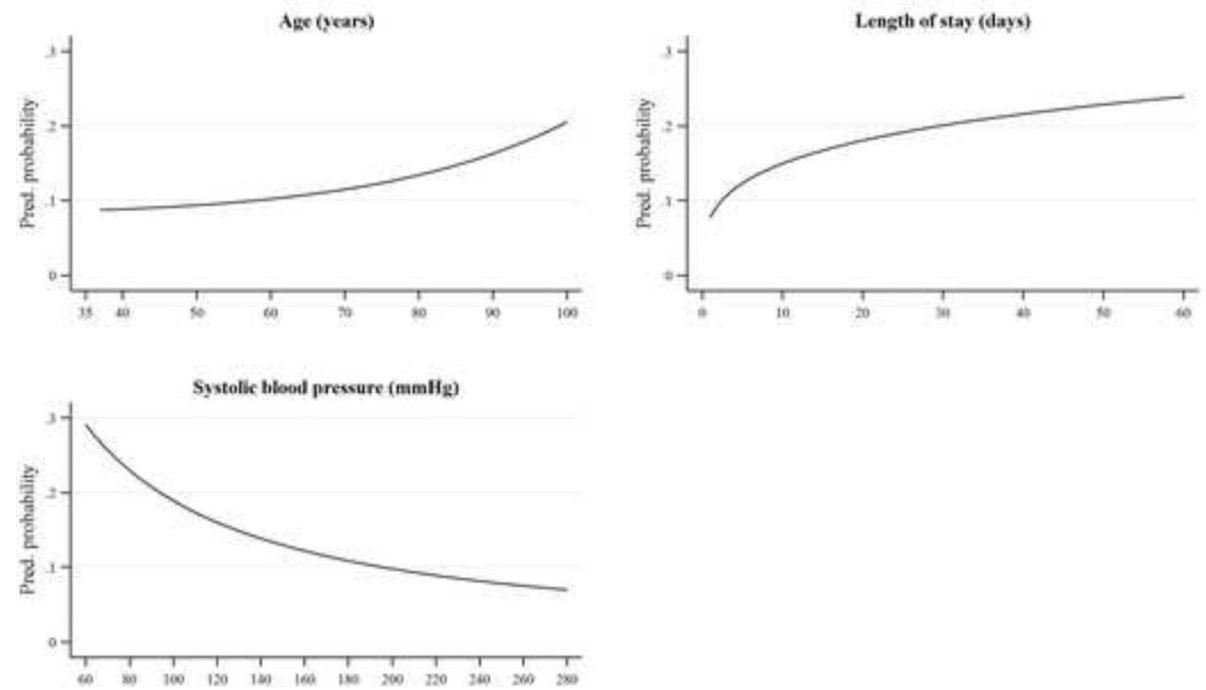

Fig. 2 Predicted probabilities of 30-day mortality and readmission for each of the continuous variables retained in the HDR-plus-clinical multivariable risk-adjustment models

with 30-day mortality. In particular, the predictors of mortality after hospital admission for HF included: atrial fibrillation, sinus rhythm with a heart rate $\geq 90 \mathrm{bpm}$, serum creatinine, and low systolic blood pressure, which also was associated with a higher risk of readmission. Atrial fibrillation is a common morbidity in HF and both conditions are associated with poor outcomes [35] and cardiovascular mortality [25]. Higher heart rate is a marker of poor cardiac function and has been associated with increased risk of mortality as an independent factor [36]. Values of serum creatinine in the range 0.8 and $1.1 \mathrm{mg} / \mathrm{dL}$, which represent good renal function, were protective for 30-day mortality after hospital admission for HF; on the contrary, alteration of serum creatinine level (a marker of renal insufficiency) is often related with HF because of common risk factors and pathogenetic mechanism and is associated with an increasing risk of morbidity and mortality [31, 37]. Lastly, low systolic blood pressure is a marker of poor cardiac output in HF and thus could identify a higher-risk patient [20]. These results demonstrate that it may be useful to collect a relatively small number of specified clinical variables from medical charts that influence 30-day mortality following HF.

The risk-adjustment models for 30-day readmission based on HDRs only and HDRs plus clinical data had a lower discrimination than the mortality models, and adding clinical data did not significantly improve the model, as highlighted by other authors [6, 14]. This result suggests that the data considered for this study, 


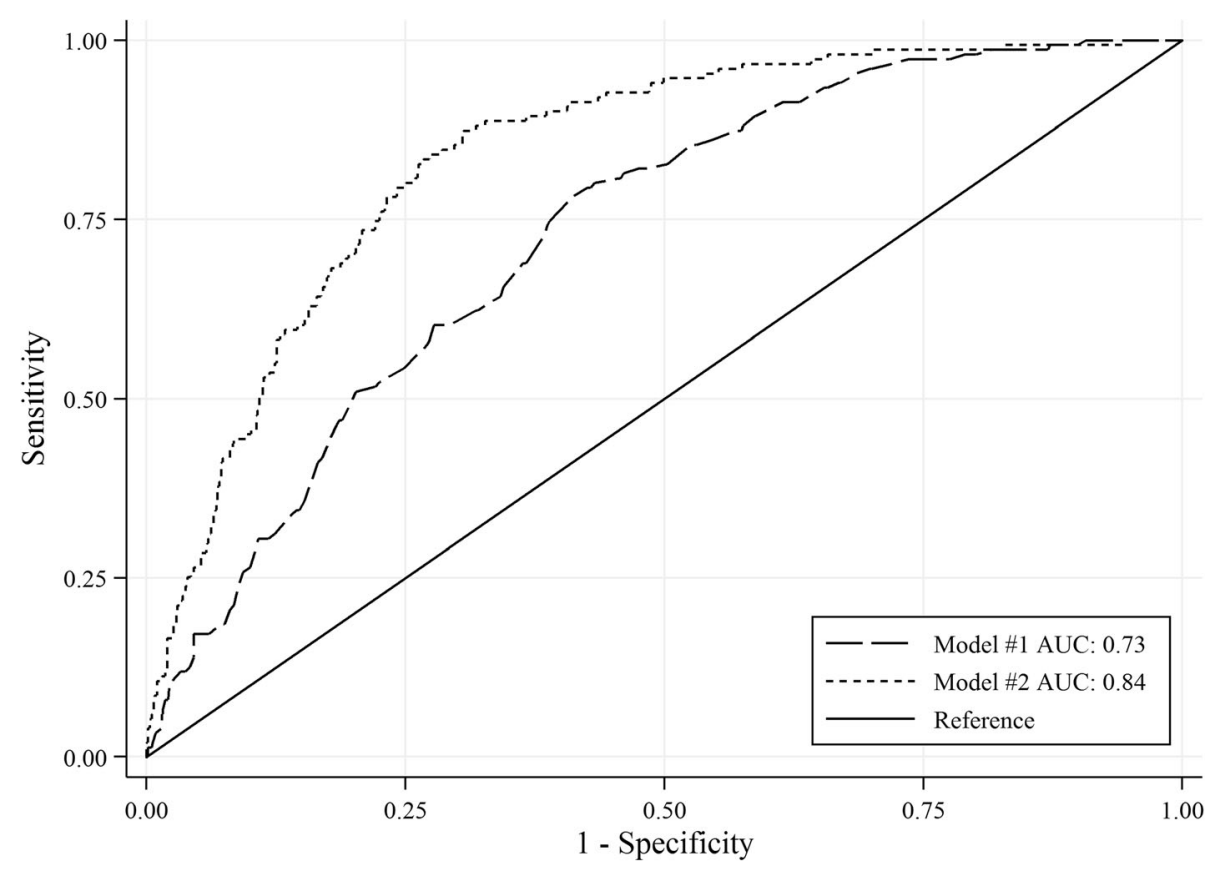

Fig. 3 ROC curves for 30-day mortality models based on HDRs only (\#1) and HDR plus clinical data (\#2). Note: The ROC curve is a plot of sensitivity versus 1 - specificity (often called the false-positive rate) that offers a summary of sensitivity and specificity across a range of cut points for a continuous predictor. The area under the curve (AUC) ranges from 0.5 (no discrimination) to a theoretical maximum of 1 (perfect discrimination). Abbreviations: ROC, receiver operation characteristic; Model \#1 AUC, area under curve for model based on HDR variables; Model \#2 AUC, area under curve for model based on HDR plus OPD plus medical charts variables

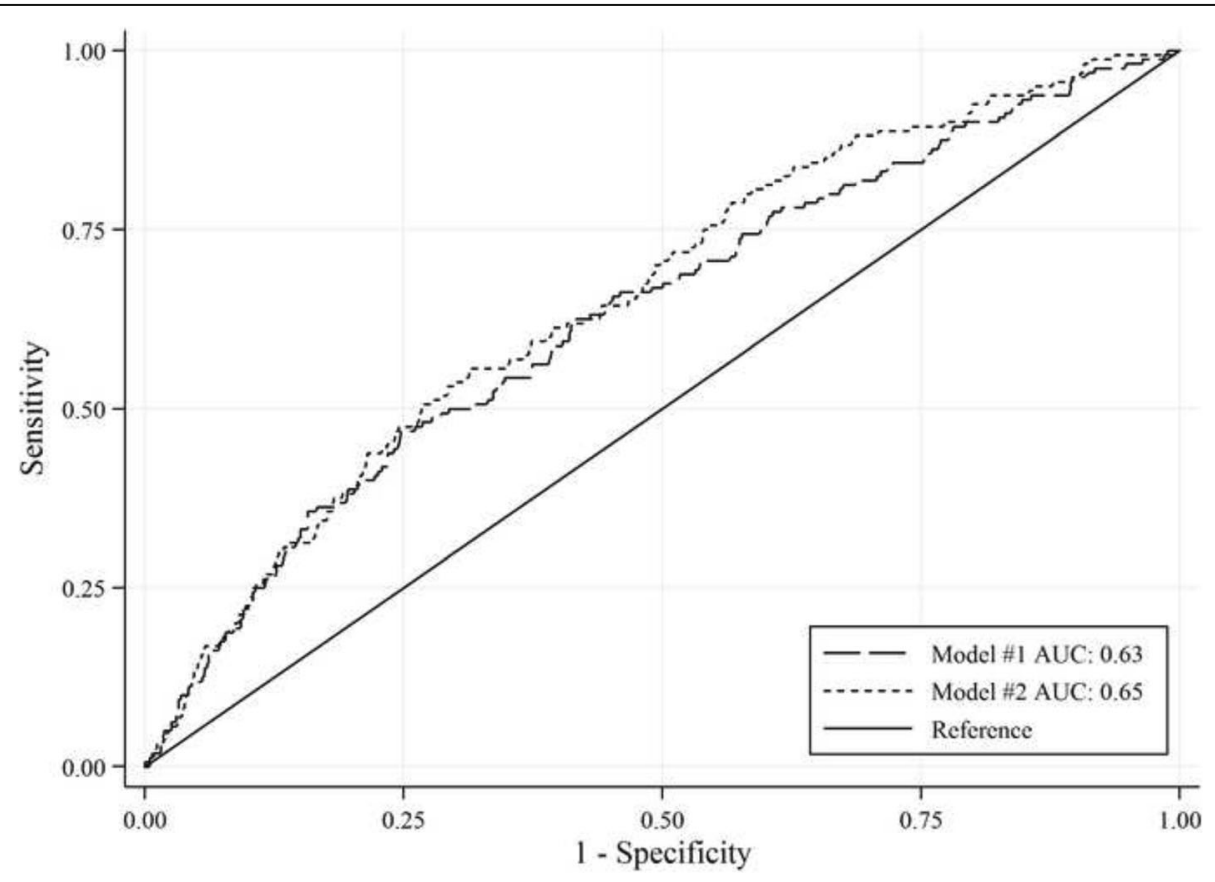

Fig. 4 ROC curves for 30-day readmission models based on HDRs only (\#1) and HDR plus clinical data (\#2) 
either administrative or clinical, did not adequately predict this outcome. A systematic review found that most of readmission risk prediction models, whether designed for comparative or clinical purposes, perform poorly, and suggested that factors associated with readmission risk may differ according to the setting and population being studied [38]. In our study, given that differences in the case mix of the study patients were not substantial, it is likely that the variables that we collected did not describe sufficiently the patient's clinical complexity, or that the discrimination of the model for readmissions depended only partially on characteristics regarding patient's severity and comorbidities. Hospital readmissions may be influenced by other factors, such as quality of care and organizational structure and processes, that were not evaluated in this study $[6,38-40]$. As an example, many studies have highlighted the effectiveness of multidisciplinary interventions pre and post discharge on readmissions of patients with $\operatorname{HF}[2,41-44]$. In order to make readmission models clinically more useful $[15,38]$, efforts are thus needed to identify variables that may play an important role in predicting this outcome.

An important finding from this study is that previous drug prescriptions had no impact on either 30-day mortality or readmission risk-adjustment models. This may reflect the fact that comorbidities derived from HDRs (either in the index hospitalization or in the previous two years) were comprehensive, and data about drug utilization reflecting the presence of these diseases did not add relevant information to the models. However, a recent study found that inpatient medications, such as insulin, antipsychotics and other drugs with many adverse effects, are associated with a higher risk of 30-day readmission [45]. This information demonstrates that efforts to extract previous drug prescriptions may not be warranted to increase the discrimination of 30-day readmission models for patients with HF, but that data on drugs used during hospital care may substantially enhance their predictive value.

The results of this study should be interpreted in light of its strengths and limitations. This is the first study conducted in Italy that evaluated the contribution of clinical variables and data on drug prescriptions in riskadjustment models for HF quality of care. Moreover, we investigated a very large number of clinical variables. Limitations include, first, the potential lack of generalizability to other settings; however, this study included all patients from one of the largest Italian LHAs and it is likely that our findings would be generalizable to other regions or countries with a population composition and healthcare delivery system similar to those of this study. Second, data recording and accuracy might have differed to some degree among the three hospitals under study, thus affecting the reliability and validity of results. Third, we did not consider some variables that have been shown to be predictive of mortality and readmission among patients with HF (e.g., body mass index, left ventricular ejection fraction, brain natriuretic peptide, etc.) [15] because they were scarcely reported in medical charts and, when present, mostly measured after medical interventions. Overall, the strengths of this study largely outweigh the limitations.

\section{Conclusions}

In the present study we identified clinical data that significantly improved the discrimination and goodness of fit of a risk-adjustment model based on HDRs to predict 30-day mortality following HF. Because the Italian Ministry of Health has planned to enhance, by 2017, the HDRs Database with the addition of clinical data for specific diseases and procedures, our results identified three "candidate" variables (i.e., systolic blood pressure, heart rhythm, and serum creatinine) that might be easily included in the HDRs to characterize the severity in patients with HF and improve the prediction of 30-day mortality.

Further research is needed to understand the contribution of some additional variables such as organizational and environmental factors, social support, substance abuse and functional status, to predicting 30-day readmissions in patients with HF.

\section{Additional files}

Additional file 1: Description of data sources. (PDF 9 kb)

Additional file 2: List of comorbidities retrieved from the Hospital Discharge Records Database. (PDF $16 \mathrm{~kb}$ )

Additional file 3: Primary data analyzed for this study. (XLS $901 \mathrm{~kb}$ )

\section{Acknowledgements}

The authors thank Silvio Candelori, MD, Emanuele Farolfi, MD, Enrica Perrone, MD, and Tiziana Sanna, MD, from the University of Bologna for collecting data from medical chart review. The authors would also like to thank Barbara Bordoni, PhD, MD, from Bellaria Hospital; Aldo Pietro Maggioni, MD, from ANMCO Research Center; and Adalgisa Protonotari, MD, from the LHA of Bologna for their assistance in writing the paper and training medical residents to abstract data from medical charts.

\section{Funding}

This study was not funded.

\section{Availability of data and materials}

All data generated or analyzed during this study are included in this published article (see Additional file 3).

\section{Authors' contributions}

$J$ conceived the study, performed the statistical analysis, interpreted the results and drafted the manuscript. VMA conceived the study, interpreted the results and drafted the manuscript. TH interpreted the results and drafted the

manuscript. CD drafted the manuscript. IC performed the statistical analysis. SU conceived the study and interpreted the results. GD conceived the study and interpreted the results. PR interpreted the results and drafted the manuscript. MPF conceived the study, interpreted the results and drafted the manuscript. All authors read and approved the final manuscript. 


\section{Competing interests}

The authors declare that they have no competing interests.

\section{Consent for publication}

Not applicable.

\section{Ethics approval and consent to participate}

The study was exempt from notification to the Ethics Committee of the LHA of Bologna because it consisted of a review of existing patients' charts (similarly to routine audit activities) and did not imply collection of additional data from patients. All patients signed a consent form at hospital admission for use of their data for administrative and research purposes, as is done routinely to comply with the privacy law, and no further consent was required. Data abstracted from medical charts were linked to HDRs and encrypted at the Regional Health Information System Office. This encryption eliminates the ability to trace the patient's identity or other sensitive data. None of the authors were involved in the treatment of the study patients and had access to any identifying information before it was encrypted by the Regional Health Information System Office.

The study was carried out in conformity with the regulations on data management of the Regional Healthcare Authority of Emilia-Romagna, and with the Italian "Code of conduct and professional practice applying to processing of personal data for statistical and scientific purposes" (Art. 20-21, DL 196/2003) (http://www.garanteprivacy.it/web/guest/home/ docweb/-/docwebdisplay/docweb/1115480) published in the Official Journal no. 190 of

August 14, 2004) which explicitly exempts the need of ethical approval for encrypted data (Preamble \#8) collected for scientific and healthcare purposes.

In Italy, anonymous administrative data-gathering is subject to the law Protection of individuals and other subjects with regard to the processing of personal data, ACT no. 675 of 31.12.1996 (amended by Legislative Decree no. 123 of 09.05.1997, no. 255 of 28.07.1997, no. 135 of 08.05.1998, no. 171 of 13.05.1998, no. 389 of 6.11 .1998 , no. 51 of 26.02 .1999 , no. 135 of 11.05 .1999 , no. 281 of 30.07.1999, no. 282 of 30.07 .1999 and no. 467 of 28.12.2001) (http://www.privacy.it/legge675encoord.html).

\section{Author details}

${ }^{1}$ Department of Biomedical and Neuromotor Sciences, Alma Mater Studiorum - University of Bologna, via San Giacomo 12, 40126 Bologna, Italy. ${ }^{2}$ Department of Surgery, Stanford University, 300 Pasteur Drive, Stanford, CA 94305-2200, USA. ${ }^{3}$ Department of Clinical Governance, Bologna Local Healthcare Authority, via Castiglione 29, 40124 Bologna, Italy. ${ }^{4}$ Department of Programming and Control, Bologna Local Healthcare Authority, via Castiglione 29, 40124 Bologna, Italy. ${ }^{5}$ Department of Cardiology, Bellaria Hospital, via Altura 3, 40139 Bologna, Italy. ${ }^{6}$ Department of Cardiology, Maggiore Hospital, Largo Nigrisoli 2, 40133 Bologna, Italy.

\section{Received: 25 November 2015 Accepted: 31 August 2016} Published online: 06 September 2016

\section{References}

1. McMurray JJ, Adamopoulos S, Anker SD, Auricchio A, Böhm M, Dickstein K et al. ESC Guidelines for the diagnosis and treatment of acute and chronic heart failure 2012: The Task Force for the Diagnosis and Treatment of Acute and Chronic Heart Failure 2012 of the European Society of Cardiology. Developed in collaboration with the Heart Failure Association of the ESC (HFA). Eur J Heart Fail. 2012;14:803-69.

2. Takeda A, Taylor SJC, Taylor RS, Khan F, Krum H, Underwood M. Clinical service organisation for heart failure. Cochrane Database Syst Rev. 2012;9:CD002752.

3. Groene O, Skau JK, Frølich A. An international review of projects on hospital performance assessment. Int J Qual Health Care. 2008;20:162-71.

4. Sasaki N, Lee J, Park S, Umegaki T, Kunisawa S, Otsubo T, et al. Development and validation of an acute heart failure-specific mortality predictive model based on administrative data. Can J Cardiol. 2013;29:1055-61.

5. Krumholz HM, Wang Y, Mattera JA, Wang Y, Han LF, Ingber MJ, et al. An administrative claims model suitable for profiling hospital performance based on 30-day mortality rates among patients with heart failure. Circulation. 2006;113:1693-701.

6. Keenan PS, Normand SL, Lin Z, Drye EE, Bhat KR, Ross JS, et al. An administrative claims measure suitable for profiling hospital performance on the basis of 30-day all-cause readmission rates among patients with heart failure. Circ Cardiovasc Qual Outcomes. 2008;1:29-37.

7. Johnston TC, Coory MD, Scott I, Duckett S. Should we add clinical variables to administrative data?: The case of risk-adjusted case fatality rates after admission for acute myocardial infarction. Med Care. 2007;45:1180-5.

8. Pine $M$, Jordan $H S$, Elixhauser $A$, Fry DE, Hoaglin DC, Jones $B$, et al. Enhancement of claims data to improve risk adjustment of hospital mortality. JAMA. 2007;297:71-6.

9. Agenzia Nazionale per i Servizi Sanitari Regionali (Agenas). Programma Nazionale Esiti. 2014. http://95.110.213.190/PNEed14_EN/index.php. Accessed 13 Nov 2015.

10. Tabak YP, Johannes RS, Silber JH. Using automated clinical data for risk adjustment: development and validation of six disease-specific mortality predictive models for pay-for-performance. Med Care. 2007:45:789-805.

11. Escobar GJ, Greene JD, Scheirer P, Gardner MN, Draper D, Kipnis P. Risk-adjusting hospital inpatient mortality using automated inpatient, outpatient, and laboratory databases. Med Care. 2008;46:232-9.

12. Tabak YP, Sun X, Derby KG, Kurtz SG, Johannes RS. Development and validation of a disease-specific risk adjustment system using automated clinical data. Health Serv Res. 2010;45:1815-35.

13. Colais P, Di Martino M, Fusco D, Davoli M, Aylin P, Perucci CA. Using clinical variables and drug prescription data to control for confounding in outcome comparisons between hospitals. BMC Health Serv Res. 2014;14:495.

14. Hammill BG, Curtis LH, Fonarow GC, Heidenreich PA, Yancy CW, Peterson $E D$, et al. Incremental value of clinical data beyond claims data in predicting 30-day outcomes after heart failure hospitalization. Circ Cardiovasc Qual Outcomes. 2011:4:60-7.

15. Rahimi K, Bennett D, Conrad N, Williams TM, Basu J, Dwight J, et al. Risk prediction in patients with heart failure: a systematic review and analysis. JACC: Heart Failure. 2014;2:440-6.

16. Fonarow GC, Adams Jr KF, Abraham WT, Yancy CW, Boscardin WJ. Risk stratification for in-hospital mortality in acutely decompensated heart failure: classification and regression tree analysis. JAMA. 2005;293:572-80.

17. Levy WC, Mozaffarian D, Linker DT, Sutradhar SC, Anker SD, Cropp AB, et al. The Seattle heart failure model: prediction of survival in heart failure. Circulation. 2006:113:1424-33.

18. Fabbri G, Gorini M, Maggioni AP. IN-CHF: il registro italiano dello scompenso cardiaco. Dieci anni di esperienza. G Ital Cardiol. 2006;7:689-94.

19. Butler J, Kalogeropoulos A, Georgiopoulou V, Belue R, Rodondi N, Garcia M, et al. Incident heart failure prediction in the elderly: the health $A B C$ heart failure score. Circ Heart Fail. 2008;1:125-33.

20. O'Connor CM, Abraham WT, Albert NM, Clare R, Gattis Stough W, Gheorghiade M, et al. Predictors of mortality after discharge in patients hospitalized with heart failure: An analysis from the Organized Program to Initiate Lifesaving Treatment in Hospitalized Patients with Heart Failure (OPTIMIZE-HF). Am Heart J. 2008;156:662-73.

21. Peterson PN, Rumsfeld JS, Liang L, Albert NM, Hernandez AF, Peterson ED, et al. A validated risk score for in-hospital mortality in patients with heart failure from the American Heart Association get with the guidelines program. Circ Cardiovasc Qual Outcomes. 2010;3:25-32.

22. Hilbe JM. Logistic Regression Models. Boca Ratona: Chapman \& Hall/CRC Press; 2009.

23. Seshan VE, Gönen M, Begg CB. Comparing ROC curves derived from regression models. Stat Med. 2013;32:1483-93.

24. Abraham WT, Fonarow GC, Albert NM, Stough WG, Gheorghiade M Greenberg BH, et al. Predictors of in-hospital mortality in patients hospitalized for heart failure: insights from the Organized Program to Initiate Lifesaving Treatment in Hospitalized Patients with Heart Failure (OPTIMIZE-HF). J Am Coll Cardiol. 2008;52:347-56.

25. Bajaj NS, Bhatia V, Sanam K, Ather S, Hashim T, Morgan C, et al. Impact of atrial fibrillation and heart failure, independent of each other and in combination, on mortality in community-dwelling older adults. Am J Cardiol. 2014;114:909-13.

26. Eapen ZJ, Greiner MA, Fonarow GC, Yuan Z, Mills RM, Hernandez AF, et al. Associations between atrial fibrillation and early outcomes of patients with heart failure and reduced or preserved ejection fraction. Am Heart J. 2014;167:369-75.

27. Zakeri R, Chamberlain AM, Roger VL, Redfield MM. Temporal relationship and prognostic significance of atrial fibrillation in heart failure patients with preserved ejection fraction: a community-based study. Circulation. 2013;128:1085-93. 
28. Bui AL, Grau-Sepulveda MV, Hernandez AF, Peterson ED, Yancy CV, Bhatt DL, et al. Admission heart rate and in-hospital outcomes in patients hospitalized for heart failure in sinus rhythm and in atrial fibrillation. Am Heart J. 2013;165:567-74.

29. Habal MV, Liu PP, Austin PC, Ross HJ, Newton GE, Wang X, et al. Association of heart rate at hospital discharge with mortality and hospitalizations in patients with heart failure. Circ Heart Fail. 2014;7:12-20.

30. Manzano L, Babalis D, Roughton M, Shibata M, Anker SD, Ghio S, et al. Predictors of clinical outcomes in elderly patients with heart failure. Eur J Heart Fail. 2011;13:528-36.

31. Metra M, Cotter G, Gheorghiade M, Dei Cas L, Voors AA. The role of the kidney in heart failure. Eur Heart J. 2012;33:2135-43.

32. Fonarow GC, Abraham WT, Albert NM, Gattis WA, Gheorghiade M, Greenberg B, et al. Organized Program to Initiate Lifesaving Treatment in Hospitalized Patients with Heart Failure (OPTIMIZE-HF): rationale and design. Am Heart J. 2004;148:43-51.

33. Gheorghiade M, Abraham WT, Albert NM, Greenberg BH, O'Connor CM, She $L$, et al. Systolic blood pressure at admission, clinical characteristics, and outcomes in patients hospitalized with acute heart failure. JAMA. 2006;296:2217-26.

34. Giamouzis G, Kalogeropoulos A, Georgiopoulou V, Laskar S, Smith AL, Dunbar S, et al. Hospitalization epidemic in patients with heart failure: risk factors, risk prediction, knowledge gaps, and future directions. J Card Fail. 2011;17:54-75

35. Wang TJ, Larson MG, Levy D, Vasan RS, Leip EP, Wolf PA, et al. Temporal relations of atrial fibrillation and congestive heart failure and their joint influence on mortality: the Framingham Heart Study. Circulation. 2003;107:2920-5.

36. Kannel WB, Kannel C, Paffenbarger Jr RS, Cupples LA. Heart rate and cardiovascular mortality: the Framingham Study. Am Heart J. 1987;113:1489-94.

37. McAlister FA, Ezekowitz J, Tonelli M, Armstrong PW. Renal insufficiency and heart failure. Prognostic and therapeutic implications from a prospective cohort study. Circulation. 2004;109:1004-9.

38. Kansagara D, Englander H, Salanitro A, Kagen D, Theobald C, Freeman M, et al. Risk prediction models for hospital readmission: a systematic review. JAMA. 2011;306:1688-98.

39. Amarasingham R, Moore BJ, Tabak YP, Drazner MH, Clark CA, Zhang S, et al. An automated model to identify heart failure patients at risk for 30-day readmission or death using electronic medical record data. Med Care. 2010;48:981-8.

40. Horwitz LI, Wang Y, Desai MM, Curry LA, Bradley EH, Drye EE, et al. Correlations among risk-standardized mortality rates and among riskstandardized readmission rates within hospitals. J Hosp Med. 2012;7:690-6.

41. Rich MW, Beckham V, Wittenberg C, Leven CL, Freedland KE, Carney RM. A multidisciplinary intervention to prevent the readmission of elderly patients with congestive heart failure. N Engl J Med. 1995;333:1190-5.

42. Garin N, Carballo S, Gerstel E, Lerch R, Meyer P, Zare M, et al. Inclusion into a heart failure critical pathway reduces the risk of death or readmission after hospital discharge. Eur J Intern Med. 2012;23:760-4.

43. Bradley EH, Curry L, Horwitz LI, Sipsma H, Wang Y, Walsh MN, et al. Hospital strategies associated with 30-day readmission rates for patients with heart failure. Circ Cardiovasc Qual Outcomes. 2013;6:444-50.

44. Avaldi VM, Lenzi J, Castaldini I, Urbinati S, Di Pasquale G, Morini M, et al. Hospital readmissions of patients with heart failure: the impact of hospital and primary care organizational factors in northern Italy. PLoS One. 2015;10:e0127796.

45. Cronin PR, Greenwald JL, Crevensten GC, Chueh HC, Zai AH. Development and implementation of a real-time 30-day readmission predictive model. AMIA Annu Symp Proc. 2014;2014:424-31.

\section{Submit your next manuscript to BioMed Central and we will help you at every step:}

- We accept pre-submission inquiries

- Our selector tool helps you to find the most relevant journal

- We provide round the clock customer support

- Convenient online submission

- Thorough peer review

- Inclusion in PubMed and all major indexing services

- Maximum visibility for your research

Submit your manuscript at www.biomedcentral.com/submit
Biomed Central 